\title{
NUMERICAL STUDY OF SHEET CAVITATION BREAK-OFF PHENOMENON ON A CASCADE HYDROFOIL
}

\author{
Yuka IGA ${ }^{1}$, Motohiko NOHMI ${ }^{2}$, Akira GOTO ${ }^{2}$, \\ Byeong Rog SHIN ${ }^{3}$, Toshiaki IKOHAGI ${ }^{3}$ \\ 1 Graduate School of Tohoku University, Sendai, 980-8579, Japan \\ ${ }^{2}$ Ebara Research Co., Ltd, Fujisawa, 251-8502, Japan \\ ${ }^{3}$ Institute of Fluid Science, Tohoku University, Sendai, 980-8577, Japan
}

\begin{abstract}
2-D unsteady cavity flows through hydrofoils in cascade which is the most fundamental element of turbomachinery are numerically calculated. In particular, attention was paid to instability phenomena of the sheet cavity in transient cavitation condition and the mechanism of break-off phenomenon was examined. A TVD MacCormack's scheme employing a locally homogeneous model of compressible gas-liquid two-phase media was applied to analyze above cavity flows. The present method permits us to treat the whole cavitating/noncavitating unsteady flow field. By analyzing numerical results in detail, it became clear that there are at least two mechanisms in the break-off phenomena of sheet cavity; one is that re-entrant jets play a dominant role in such a break-off phenomenon, and the other is that pressure waves propagating inside the cavity bring about an another type of break-off phenomenon accompanied with cavity surface waves.
\end{abstract}

\section{Introduction}

Cavitation is usually observed in high-speed liquid flows around such obstacles as impeller of fluid machinery. It is well known that cavity flow causes vibration, noise, damage and decline of efficiency in hydraulic systems. The improvement of predictability of cavity flow is a common subject to designers of the high-speed fluid machinery. In recent years, large-scale computations become possible with the enlargement and speedup of computers. So, it is expected to improve the prediction technique of cavity flow using numerical simulation. However, because cavity flows include complex and strong unsteady phenomena with phase change, fluid transients, vortex shedding and turbulence, mathematical modeling and numerical method of such high-speed gas-liquid two-phase flows seem considerably difficult to establish. Several numerical models have been proposed so far by Deshpande, Feng and Merkle(1994), Chen and Heister(1995) and Matsumoto, Kanbara and Sugiyama(1998), and their validity and applicability are being examined for some kinds of cavity flows.

In this study, a numerical method based on a locally homogeneous model of compressible gas-liquid twophase media (see, for example, Shin and Ikohagi(1999)) is applied to cavity flow through cascade hydrofoils at a stator condition(see, for example, Watanabe and Tsujimoto(1998)). A particular attention was paid to break-off phenomenon (see, for example, Le, et al.(1993), Hofmann, et al.(1999)) of the sheet cavity in transient cavitation condition and the mechanism was examined for some cascade arrangements.

\section{Numerical Method for Cavity Flow}

\subsection{Mathematical Modeling}

In the present mathematical model, the gas-liquid two-phase mixture inside the cavity is treated as a locally homogeneous pseudo-single phase media with an apparent compressibility. By approximating the gas-liquid mixture of any finite bubbles existing in each control volume to that of infinite number of infinitesimal bubbles having the same void fraction, the local mixture condition of cavity is specified in each computational cell. Since this molding can be applied to the inside mixture fluid of cavity similar to the outside single phase fluid, it is possible to analyze macroscopically the strong unsteady and complex cavity flows. The equation 
of state for a locally homogeneous gas-liquid two-phase media can be expressed as follows by using quality Y (mass fraction of gas phase),

$$
\rho=\frac{p\left(p+p_{c}\right)}{K(1-Y) p\left(T+T_{0}\right)+R Y\left(p+p_{c}\right) T}
$$

where $\rho, p, T$ are the mixture density, pressure, temperature of the two-phase media, respectively. And $p_{c}, T_{0}$ are the pressure and temperature constants of liquid, and $R, K$ are the gas and liquid constants, respectively. Since the temperature change of the usual cavity flow is negligible enough to suppose an isothermal condition, the isothermal speed of sound $c$ is expressed as follows,

$$
c^{2}=\frac{p\left(p+p_{c}\right)}{\rho} \times \frac{Y R\left(p+p_{c}\right)+(1-Y) K p}{Y\left\{R\left(p+p_{c}\right)^{2}-K p^{2}\right\}-\rho K R Y(1-Y) p_{c} T_{0}+K p^{2}}
$$

Also, the next relation is employed between void fraction $\alpha$ and quality $Y$.

$$
\rho(1-Y)=(1-\alpha) \rho_{l} \quad \rho Y=\alpha \rho_{g}
$$

It was confirmed that the present speed of sound $c$ agrees well with the experimental data given by Karplus at $20^{\circ} \mathrm{C}$. And, the mixture viscosity coefficient is approximated as, $\mu=(1-\alpha)(1+2.5 \alpha) \mu_{l}+\alpha \mu_{g}$, where the subscripts $g, l$ mean the states of gas and liquid phases, respectively.

\subsection{Fundamental Equations}

The governing equations for above-mentioned two-phase media are the 2-D compressible Navier-Stokes equations to which the mass conservation law of the gas phase is added.

$$
\frac{\partial \boldsymbol{Q}}{\partial t}+\frac{\partial\left(\boldsymbol{E}-\boldsymbol{E}_{v}\right)}{\partial \xi}+\frac{\partial\left(\boldsymbol{F}-\boldsymbol{F}_{v}\right)}{\partial \eta}=0
$$

where the unknown variable vector $\boldsymbol{Q}$, the flux vectors $\boldsymbol{E}, \boldsymbol{F}$ and the viscous terms $\boldsymbol{E}_{v}, \boldsymbol{F}_{v}$ are as follows,

$$
\boldsymbol{Q}=\frac{1}{J}\left(\begin{array}{c}
\rho \\
\rho u \\
\rho v \\
\rho Y
\end{array}\right), \boldsymbol{E}=\frac{1}{J}\left(\begin{array}{c}
\rho U \\
\rho u U+\xi_{x} p \\
\rho v U+\xi_{y} p \\
\rho U Y
\end{array}\right), \boldsymbol{F}=\frac{1}{J}\left(\begin{array}{c}
\rho V \\
\rho u V+\eta_{x} p \\
\rho v V+\eta_{y} p \\
\rho V Y
\end{array}\right), \boldsymbol{E}_{v}=\frac{1}{J}\left(\begin{array}{c}
0 \\
\xi_{x} \tau_{x x}+\xi_{y} \tau_{x y} \\
\xi_{x} \tau_{y x}+\xi_{y} \tau_{y y} \\
0
\end{array}\right), \boldsymbol{F}_{v}=\frac{1}{J}\left(\begin{array}{c}
0 \\
\eta_{x} \tau_{x x}+\eta_{y} \tau_{x y} \\
\eta_{x} \tau_{y x}+\eta_{y} \tau_{y y} \\
0
\end{array}\right)
$$

In Eq.(2), $u, v$ are velocity components in Cartesian coordinates $(x, y), \tau$ is viscous stress tensor, $J=$ $\xi_{x} \eta_{y}-\eta_{x} \xi_{y}$ is Jacobian and $U, V$ are contravariant velocity components in curvilinear coordinates $(\xi, \eta)$.

\subsection{Numerical Method}

In this study, unsteady cavity flows are simulated solving the above governing equations using finite difference method. Also, it is necessary to simulate stably the high-speed cavity flows with discontinuities like a gas-liquid interface which has large density jump, so that TVD MacCormack's scheme (see, for example, Yee(1987)) having 2nd order accuracy of time and space was used. Furthermore, time splitting method was used in order to improve the stability of the solution in multi-dimensional problem.

\section{Results and Discussion}

\subsection{Validation of Present Method (Comparison with experimental results)}

The present numerical method is considered relevant to the analysis of complex cavity flows, because it can treat the whole flow fields at once. On the other hand, as the compressible numerical scheme is used, a socalled noncavitating flow is handled approximately as a low Mach number flow. Therefore, the comparison of numerical result with experimental one under cavitating and noncavitating conditions was made, and the present method was validated through the comparison.

Cavity flows around a single hydrofoil and hydrofoils in cascade are taken up. The hydrofoil geometry is ClarkY of thickness ratio $11.7 \%$ whose performance data are available comparatively. Computations were performed using a $\mathrm{H}$ typed grid which can compose a cascade passage geometry cyclically. In case of single hydrofoil, a pitch $(t)$, chord $(c)$ ratio was set as $t / c=20$ where a single hydrofoil condition is fulfilled approximately. And in case of cascade hydrofoil, a cascade arrangement of pitch/chord ratio $t / c=0.9$ and stagger angle $\gamma=30^{\circ}$ was selected. The stator condition of constant angle of attack and the constant total pressure condition were applied to inlet boundary, and the constant pressure condition was applied to 

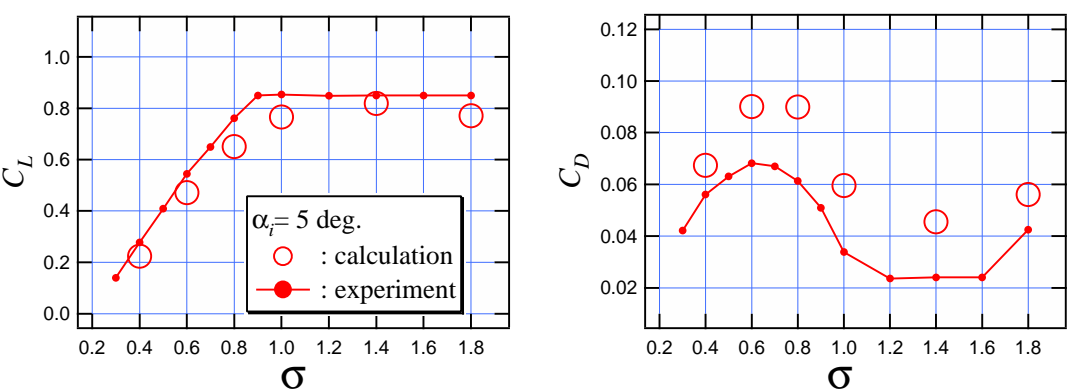

Figure 1: Time-averaged lift and drag coefficients of a single hydrofoil at several cavitation numbers (ClarkY $11.7 \%)$
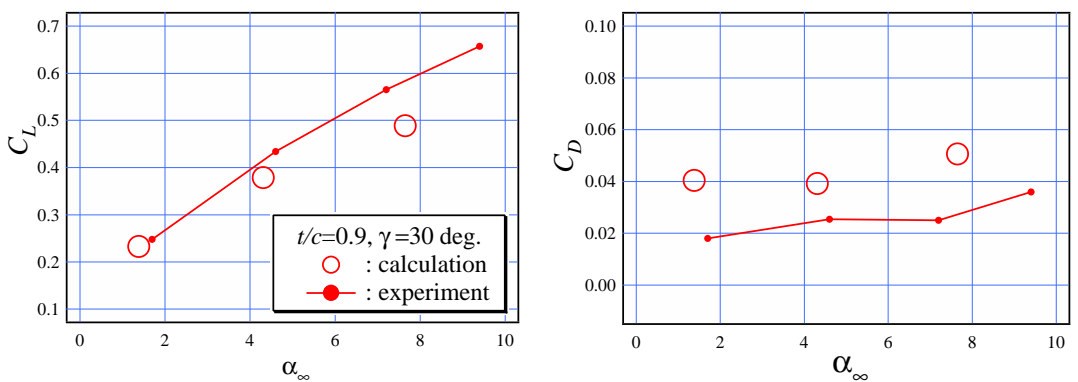

Figure 2: Time-averaged lift and drag coefficients of cascade hydrofoils (ClarkY 11.7\%, noncavitating state)

outlet boundary. Furthermore, the cyclic boundary condition between each cascade passage and the non-slip condition on hydrofoil surface were imposed.

First, cavitating flows around single hydrofoil (in fact, $t / c=20, \gamma=0^{\circ}$ ) were analyzed for various cavitation number $\sigma$ and an angle of attack $\alpha_{i}=5^{\circ}$. At this time, an inlet flow velocity is $U_{0}=10 \mathrm{~m} / \mathrm{s}$ and a Reynolds number is $R_{e}=7.0 \times 10^{5}$. Time-averaged lift and drag coefficients $C_{L}, C_{D}$ were calculated from numerical results, and the comparison with experimental data at the same Reynolds number(see, for example, Numachi, Tsunoda and Chida(1949)) is shown in Fig.1. Generally, it is known that $C_{L}$ decreases largely when $\sigma$ becomes smaller than a certain critical value (that is so-called cavitation break-down). The numerical result can predict a qualitative tendency of $C_{L}$ and $C_{D}$ against $\sigma$, although quantitative differences are seen to some extent especially for $C_{D}$.

Next, noncavitating cascade flows for $U_{0}=4.3 \mathrm{~m} / \mathrm{s}$ and $R_{e}=5.0 \times 10^{5}$ were calculated while the angle of attack $\alpha_{\infty}$ based on geometrical mean velocity was changed as $1.4^{\circ}, 4.3^{\circ}$ and $7.6^{\circ}$. Time-averaged lift and drag coefficients were calculated from numerical results, and the comparison with the wind tunnel experiment is shown in Fig.2. The numerical results can show a qualitative tendency even though $C_{L}$ and $C_{D}$ values tend to be somewhat overestimated and underestimated, respectively. Furthermore, the pressure distributions on hydrofoil surface for these three $\alpha_{\infty}$ are shown in Fig.3, where the experimental results are also shown for comparatively near $\alpha_{\infty}$ values. These numerical results coincide well with the experimental tendency of the surface pressure distribution. Therefore, it is confirmed that the present numerical method is fairly effective in the evaluation of the time-averaged characteristics of single and cascade hydrofoils from noncavitation to cavitation conditions.
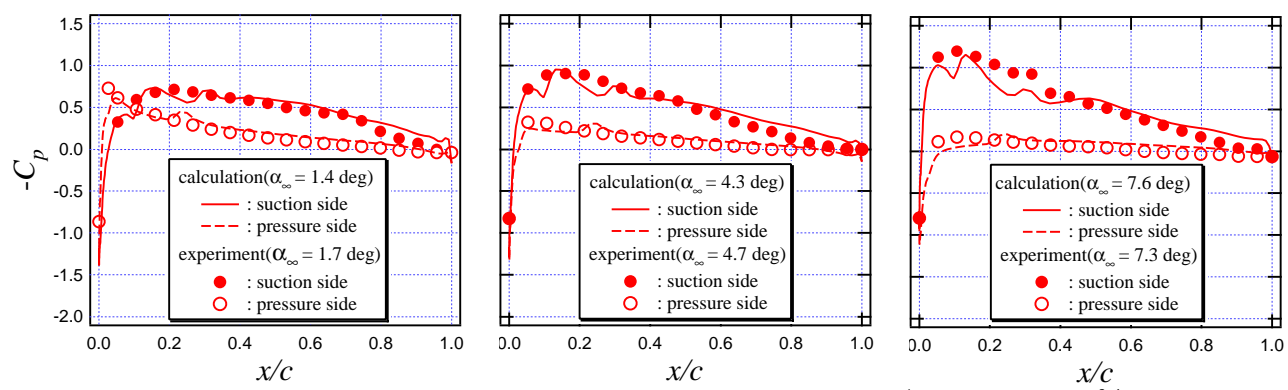

Figure 3: Time-averaged pressure distribution of cascade hydrofoils (ClarkY $11.7 \%$, noncavitating state) 

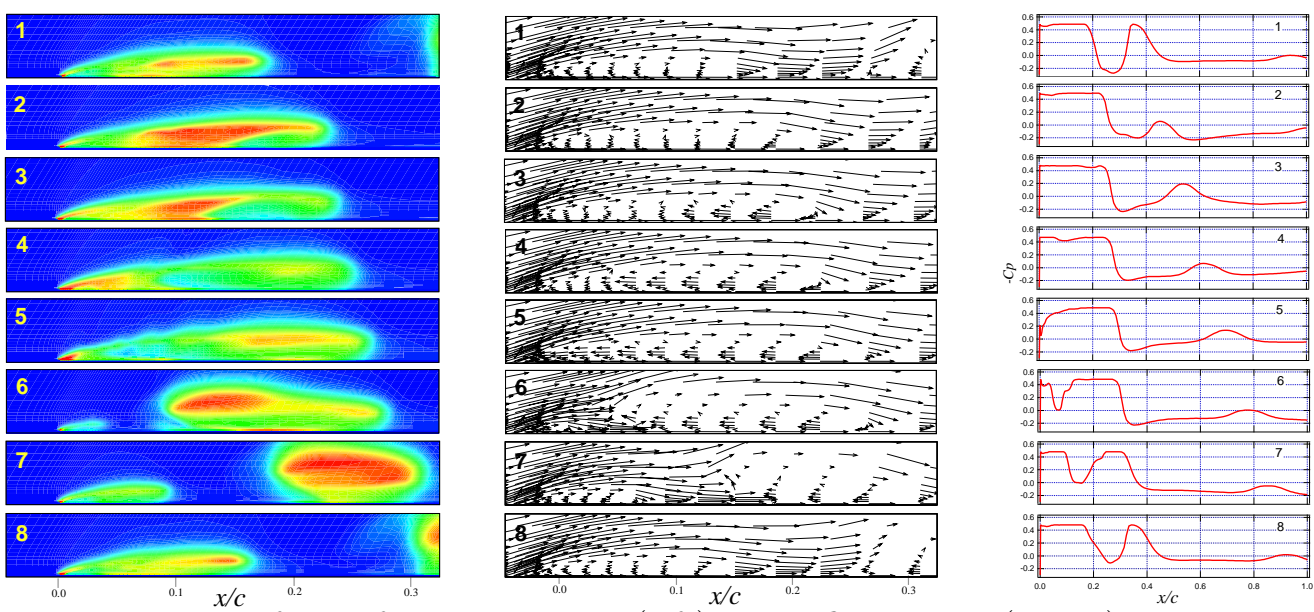

Figure 4: Time evolution of void fraction contours(left), mass flux vectors(center) and pressure distribution(right) around flat plate cascade hydrofoils $\left(t / c=0.9, \gamma=30^{\circ}, \sigma / 2 \alpha_{i}=1.95\right.$, time interval $\left.=1.2 \mathrm{~ms}\right)$

\subsection{Sheet Cavitation Break-off Phenomenon}

Numerical analysis of the aspect of cavity flow was carried out by taking up the unsteady flow through flat plate cascade which is closely related to that of inducer. In particular, attention was paid to the break-off phenomena of the sheet cavity in transient cavitation condition where the unsteadiness becomes quite strong. The stator condition of constant angle of attack $\alpha_{i}$ and the constant total pressure condition were applied to inlet boundary. Furthermore, the cyclic boundary condition between each cascade passage and the non-slip condition on hydrofoil surface were also imposed.

First of all, the transient cavitating flow through the flat plate cascade $\left(t / c=0.9, \gamma=30^{\circ}\right)$ was analyzed for $\sigma / 2 \alpha_{i}=1.95$ and $\alpha_{i}=7^{\circ}$, where the maximum cavity length $l_{\max }$ to the chord length is about $l_{\max } / c=$ 0.28 . Time evolution of void fraction, mass flux vectors and pressure distributions on the suction side of flat plate cascade hydrofoil is shown in Fig.4. The mass flux vectors are employed to distinguish between separated flow and re-entrant jet inside the cavity. A sheet cavitation formed from leading edge on suction surface(Fig.4-1) is developing toward trailing edge(Fig.4-2). Then, the re-entrant jet is forced to occur by the steep adverse pressure gradient near the cavity termination, and the re-entrant jet with a finite width moves toward the leading edge of sheet cavity(Fig.4-3,4). In the vicinity of leading edge, the jet impinges on the cavity surface layer, whose thickness becomes smaller than the jet width. At this moment, a rapid pressure increase occurs around the impinging position near the leading edge of cavity by the water hammer effect, as described later. Further, a certain surface wave is also observed on cavity surface near the leading edge(Fig.4-5). After that, the sheet cavity is beginning to break off from suction surface (Fig.4-6), and finally cavity is detached from the suction surface and shedding toward downstream (Fig.4-7). At this time, another sheet cavitation has already begun to develop in a similar manner (Fig.4-8). Thus, a break-off phenomenon due to re-entrant jet formation can be clearly simulated. Now, the pressure jump on the suction surface near the impinging position $(x / c=0.01, y / c=0.001)$ in Fig.4-5 is about $\Delta C_{p}=0.42$. Generally a water hammer pressure increase may be $\Delta p_{w}=k \times \rho c \Delta u(0.5 \leq k \leq 1)$. In this case, the water hammer pressure coefficient was evaluated as $0.32 \leq \Delta C_{p w} \leq 0.64$ from the present numerical data. Above $\Delta C_{p}$ value is in this range. On the other hand, the stagnation pressure coefficient by the primary re-entrant jet at same position was estimated as $\Delta C_{p s}=0.21$. This value does not correspond to $\Delta C_{p}$ value. Therefore, the pressure jump is considered to be brought by the water hammer action of the re-entrant jet.

The time evolution of the cavity length, the tip position of re-entrant jet and the lift coefficient are shown in Fig.5. Roughly speaking, the lift begins to increase gradually after sheet cavity breaks off from suction surface and decrease during cavity shedding to downstream, so that the lift is fluctuating with the break-off behavior. The most accelerated re-entrant jet velocity becomes $V r=3.5 \mathrm{~m} / \mathrm{s}$. Since the average inlet velocity is $10.8 \mathrm{~m} / \mathrm{sec}$, the re-entrant jet velocity is about $30 \%$ of the inlet one. Also, the frequency of cavity shedding is $114 \mathrm{~Hz}$, so that Strouhal number $\left(S_{t}\right)_{c}$ of cavity shedding based on chord length is about 0.75 and Strouhal number $\left(S_{t}\right)_{l}$ based on maximum cavity length is 0.21 . In connection with this argument, the power spectrum 

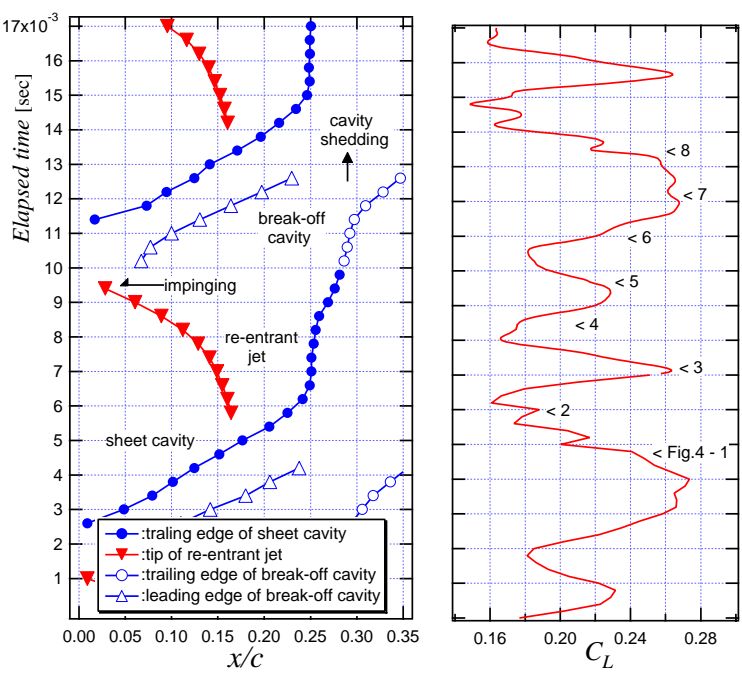

Figure 5: Time evolution of cavity lengths, re-entrant jet and lift coefficient $\left(t / c=0.9, \gamma=30^{\circ}, \sigma / 2 \alpha_{i}=1.95\right)$

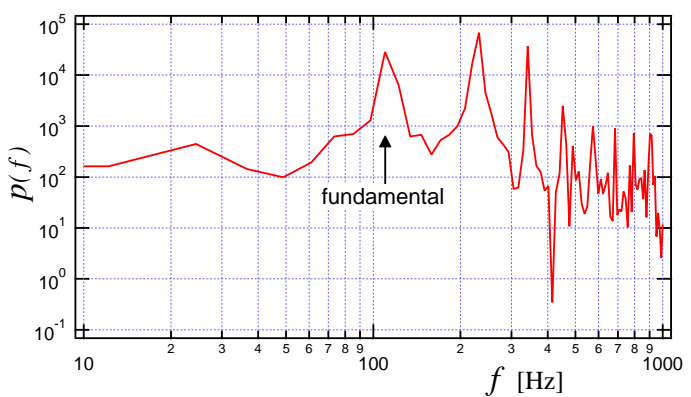

Figure 6: Power spectrum of pressure at cascade throat center $\left(t / c=0.9, \gamma=30^{\circ}, \sigma / 2 \alpha_{i}=1.95\right)$

of the pressure in the center of cascade throat have the peak at $f=110 \mathrm{~Hz}$. It is understood therefore that the break-off phenomenon is repeated quite regularly because of the evident peak. However, it should be noted that the break-off phenomenon does not occur in the case of $\alpha_{i}=3^{\circ}$ and $1.29 \leq \sigma / 2 \alpha_{i} \leq 2.10$, in which the stable attached cavity was formed in the present simulation.

Next, the transient cavitating flow $\left(\sigma / 2 \alpha_{i}=1.27, \alpha_{i}=3^{\circ}\right)$ through the flat plate cascade $(t / c=0.5, \gamma=$ $75^{\circ}$ ) as shown in Fig.7 was analyzed. In this cascade arrangement, stagger angle is larger, pitch/chord ratio is smaller and the cascade interference becomes more intense than the previous cascade one. In this case, the maximum cavity length is about $l_{\max } / c=0.43$, and the trailing edge of cavity is located in the front of cascade throat. Time evolution of void fraction on suction side of cascade hydrofoils are shown in Fig.8. The break-off phenomenon is observed even in this case. However, no evident re-entrant jet does occur like the previous case. As shown in Fig.9, pressure fluctuations can be seen in hydrofoil surface inside the cavity at each corresponding time in Fig.8. By plotting these fluctuations carefully in Fig.10, it is recognized that pressure waves are propagating inside the cavity, and one of the waves (break-off wave) separates the second half part of the sheet cavity. Also, the local angle of attack $\alpha_{\text {local }}$ near the leading edge and local throat pressure $P_{t}$ at the center of cascade throat are shown in this figure. When break-off cavity passes in cascade throat, the throat width becomes narrow (see Fig.7). Then, the liquid flow accelerates locally and the pressure in the cascade throat decreases. After the break-off cavity sheds away, the throat pressure recovers.

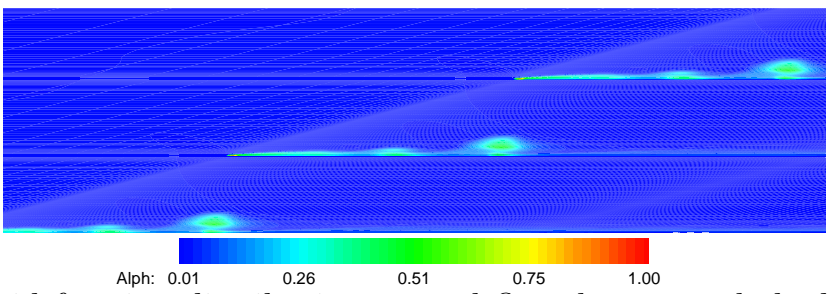

Figure 7: Instantaneous void fraction distribution around flat plate cascade hydrofoils $\left(t / c=0.5, \gamma=75^{\circ}\right.$, $\left.\sigma / 2 \alpha_{i}=1.27\right)$ 

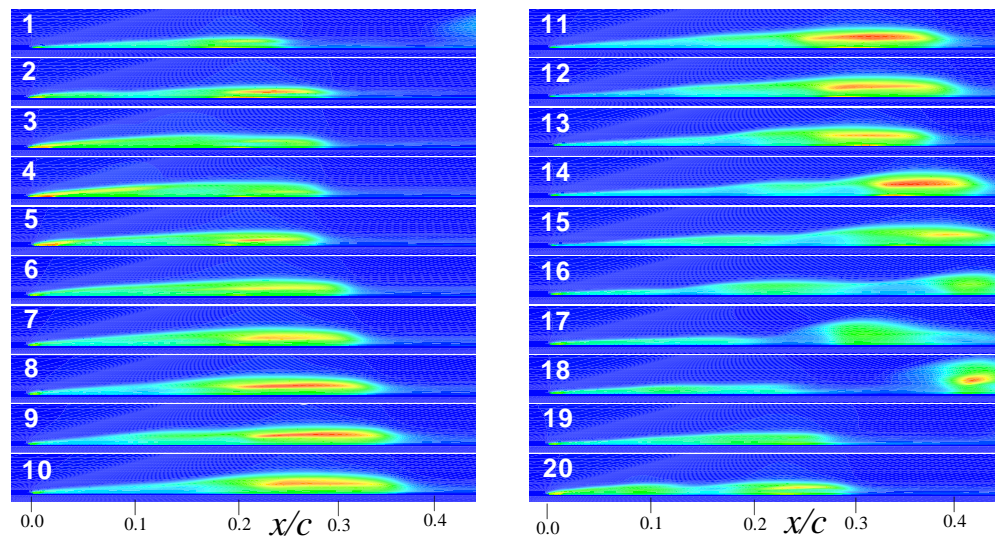

Figure 8: Time evolution of void fraction contours around flat plate cascade hydrofoils $\left(t / c=0.5, \gamma=75^{\circ}\right.$, $\sigma / 2 \alpha_{i}=1.27$, time interval $\left.=2.0 \mathrm{~ms}\right)$
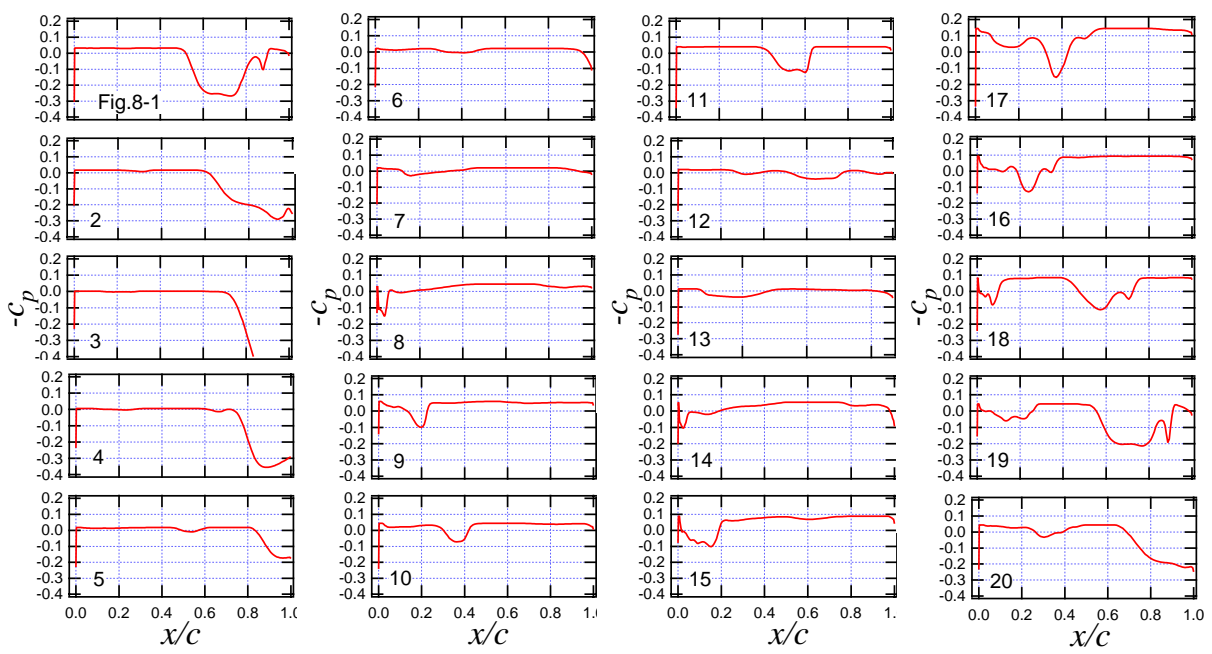

Figure 9: Time evolution of pressure distribution on suction side of flat plate cascade hydrofoils $(t / c=0.5$, $\gamma=75^{\circ}, \sigma / 2 \alpha_{i}=1.27$, time interval $\left.=2.0 \mathrm{~ms}\right)$

Such pressure fluctuations cause a great variation in the local angle of attack of a neighbor hydrofoil. Further, this rapid change of the local angle of attack causes the cavity thickness fluctuation near the leading edge, and at the same time pressure waves are produced one after another from the leading edge of cavity to the inside of the cavity. Finally, the cavity tends to break off by the instability of the cavity surface associated with these pressure wave propagations. It is considered that the break-off phenomenon is repeated with such a mechanism in this case. At this time, the cavity shedding frequency is about $29.6 \mathrm{~Hz}$, so that $\left(S_{t}\right)_{c}$ is about 0.24 and $\left(S_{t}\right)_{l}$ is 0.11 . On the other hand, in the power spectrum of the cascade throat pressure shown in Fig.11, the power spectrum near a range of $f=30 \mathrm{~Hz}$ is a little bit high, although an evident peak is not clear. So, it is understood that this type of break-off phenomenon is repeated somewhat irregularly.

Further, the transient cavitating flow $\left(\sigma / 2 \alpha_{i}=0.23, \alpha_{i}=5^{\circ}\right)$ in the same cascade arrangement was analyzed. In this case, the maximum cavity length is about $l_{\max } / c=1.1$, and the trailing edge of cavity is located in the rear of cascade throat as shown in Fig.12. Simular to the previous case, but the evident reentrant jet does not occur and the pressure waves can be seen inside the cavity. So, the break-off phenomenon seems to be caused by the same mechanisms as the previous case. Here, as shown in Fig.12-1, small cavity occurs on the pressure side of hydrofoil in this case. This is because the passage distance of the cavity surface and the pressure side of the neighbor hydrofoil became narrow by the large fluctuation of cavity volume, the local pressure rapidly decreases to cavitate slightly. And even in this case, the break-off phenomenon is repeated irregularly.

Strouhal numbers of cavity shedding are shown in Fig.13, where the blue marks are the case of re-entrant jet dominant break-off phenomenon $\left(t / c=0.9, \gamma=30^{\circ}\right)$ and the red marks are the case of pressure wave dominant break-off phenomenon $\left(t / c=0.5, \gamma=75^{\circ}\right)$. In the former case, $\left(S_{t}\right)_{c}$ based on the chord length 

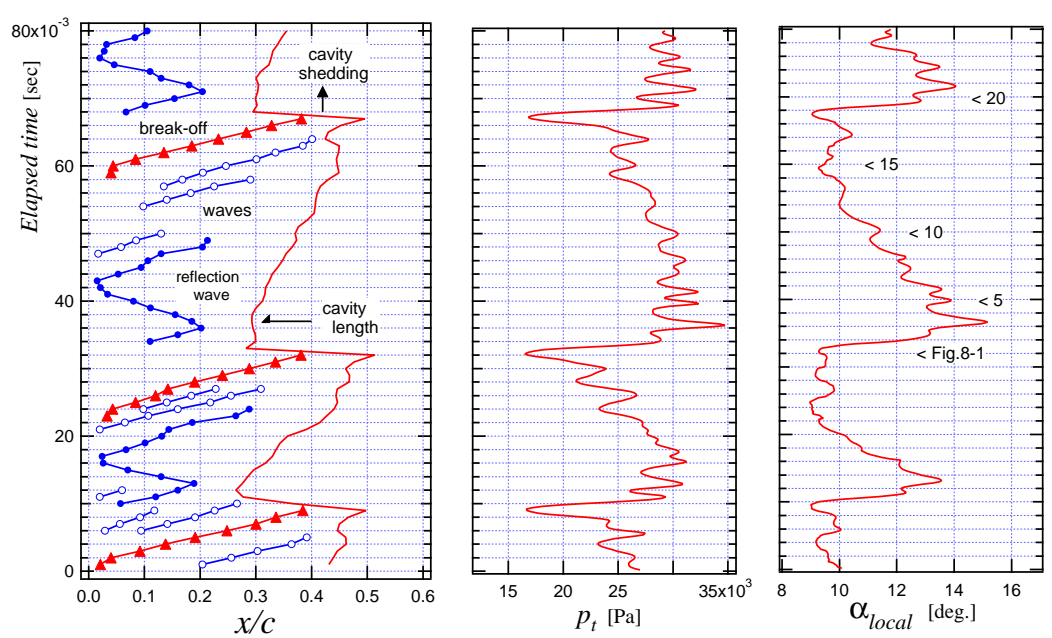

Figure 10: Time evolution of cavity length and waves in cavity $\left(t / c=0.5, \gamma=75^{\circ}, \sigma / 2 \alpha_{i}=1.27\right)$

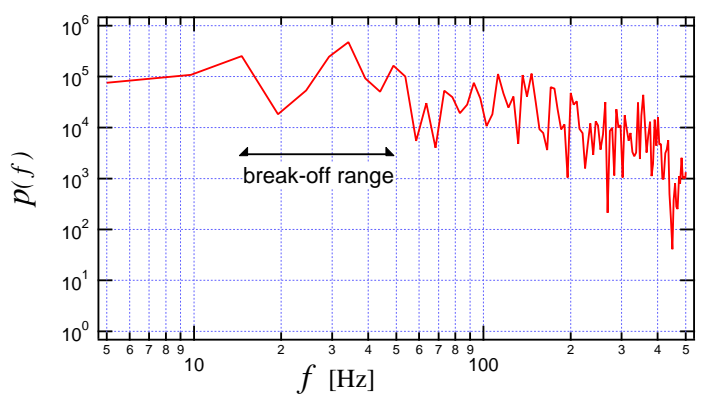

Figure 11: Power spectrum of pressure at cascade throat center $\left(t / c=0.5, \gamma=75^{\circ}, \sigma / 2 \alpha_{i}=1.27\right)$

increases with $\sigma / 2 \alpha_{i}$, while $\left(S_{t}\right)_{l}$ based on maximum cavity length are almost constant 0.2 without depending on $\sigma / 2 \alpha_{i}$ (see, for example, Le, Franc and Michel(1987)). On the other hand, in the latter case, both $\left(S_{t}\right)_{c}$ and $\left(S_{t}\right)_{l}$ values are scattering from 0.2 to 0.3 because of the somewhat irregular break-off nature.

\section{Conclusions}

The present numerical method based on a locally homogeneous model of compressible gas-liquid two-phase media has been validated to be fairly effective in the evaluation of the time-averaged characteristics of single and cascade hydrofoils from noncavitating to cavitating flows by comparison of numerical and experimental results. And, analyzing cavity flows through hydrofoils in cascade under several cavitation conditions, it is
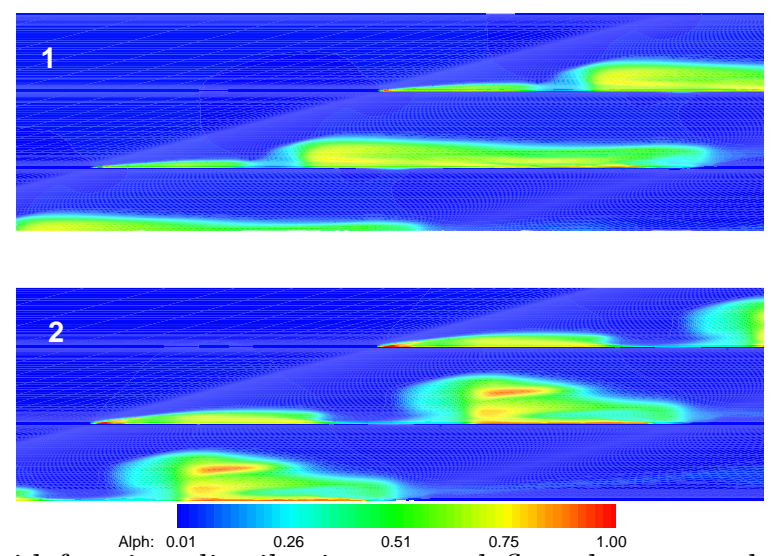

Figure 12: Instantaneous void fraction distribution around flat plate cascade hydrofoils $(t / c=0.5, \gamma=$ $75^{\circ}, \sigma / 2 \alpha_{i}=0.23$, time interval $\left.=4.0 \mathrm{~ms}\right)$ 


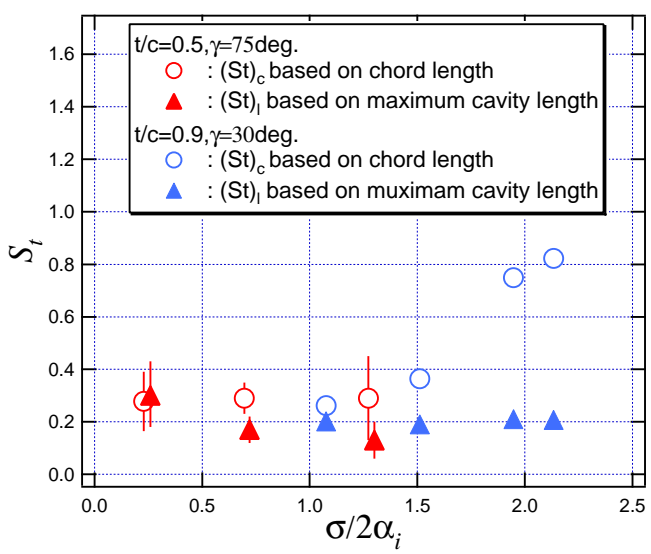

Figure 13: Strouhal number of cavity shedding on flat plate cascade hydrofoil

indicated that the present numerical method has an ability to simulate well strong unsteady cavity flows. The main results obtained from this study about the break-off phenomenon of sheet cavity on cascade hydrofoil are summarized as follows;

1. It becomes clear that there are at least two mechanisms in the break-off phenomena of sheet cavity, one is that re-entrant jets play a dominant role in a break-off phenomenon, and the other is that pressure waves propagating inside the cavity bring about an another type of break-off phenomenon accompanied with cavity surface waves.

2. In the case of re-entrant jet dominant break-off phenomenon, the jet impingement on cavity surface layer is repeated quite regularly, and the pressure jump on the hydrofoil suction surface near the impinging position is brought by the water hammer action of the re-entrant jet. However, such a break-off phenomenon does not occur in the case of smaller $\alpha_{i}$ in which the stable attached cavity is formed.

3. In the case of pressure wave dominant break-off phenomenon, the cavity tends to break off by the instability of cavity surface layer associated with pressure wave propagation. The pressure waves are produced by the cavity thickness fluctuation near the leading edge, which follows the time variation of local angle of attack due to the throat area variation caused by break-off cavity shedding. This type of break-off phenomenon is repeated somewhat irregularly.

\section{References}

Chen,Y. and Heister,S.D. (1995). Computers and Fluids, 24-7, 799-809.

Deshpande,M., Feng,J. and Merkle,C.L. (1994). J. Fluids Eng., Trans.ASME, 116, 36-44.

Hofmann,M., et al. (1999). ASME Paper FEDSM, 99-6755, 99-7259, San Francisco.

Kikuchi,H. (1959). Mem. Inst. High Sp. Mech., Tohoku Univ., 14(139), 193-218, (in Japanese).

Le,Q., Franc,J.P. and Michel,J.M. (1993). J. Fluids Eng., Trans. ASME, 115, 243-248.

Matsumoto,Y., Kanbara,T. and Sugiyama,K. (1998). ASME, PVP-377-2, 243-248.

Numachi,F., Tsunoda,H. and Chida,I. (1949). Rep. Inst. High Sp. Mech., Tohoku Univ., 1(1), 1-16, (in Japanese).

Shin,B.R. and Ikohagi,T. (1999). ASME Paper FEDSM, 99-7215, San Francisco.

Watanabe,S., Tsujimoto,Y., Franc,J.P. and Michel,J.M. (1998). Proc. 3rd Int. Symp. on Cavitation, 347-352, Grenoble, France.

Yee,H.C. (1987). NASA-TM, 89464. 